\title{
Relation entre quelques caractères morphologiques et la sensibilité à la verse en végétation du maïs (Zea mays L)
}

\author{
S Pellerin, R Trendel, A Duparque \\ avec la collaboration technique de A Blatz et B Petit \\ INRA, laboratoire d'agronomie, 68021 Colmar, France
}

(Reçu le 12 décembre 1989; accepté le 12 mai 1990)

\begin{abstract}
Résumé - On a étudié sur 12 hybrides demi-précoces les relations entre quelques caractères morphologiques et les résultats d'une verse intervenue 2 jours après les mesures, au stade 13-15 feuilles visibles. Les variables mesurées étaient le stade des plantes (nombre de feuilles visibles et étalées), la matière sèche aérienne et le nombre de racines adventives émises par entrenœud. Les notations de verse ont été effectuées sur 50 plantes par parcelle élémentaire ( 2 rangs sur 5 mètres) en essai bloc à 3 répétitions. Les plantes ont été distribuées par classe d'angle d'inclinaison par rapport à la verticale. Pour chaque hybride, on a calculé un angle moyen d'inclinaison. Vingt-quatre pieds versés ont été prélevés pour une observation détaillée des zones de rupture. Sept des 12 hybrides étudiés ont versé. La verse s'est traduite par une inclinaison des plantes depuis leur base, sans cassure de tige. L'observation détaillée de pieds versés montre l'existence de coudes ou de ruptures du cortex sur la partie basale des racines adventives des derniers entrenœuds, voire au niveau de leur point d'insertion sur la tige. On observe une corrélation négative $(r=-0,677)$ entre l'importance de la verse appréciée au travers de l'angle moyen d'inclinaison et le nombre d'entrenœuds portant des racines allongées lors du prélèvement précédant la verse. Dans le cadre d'un travail visant à rechercher des caractères liés à la résistance à la verse, ce résultat souligne la nécessité d'étudier la cinétique d'apparition des racines adventives. De même, la résistance à la rupture de ces racines et les caractéristiques morphologiques ou biochimiques qui en sont à l'origine devraient être considérées.
\end{abstract}

Zea mays / verse en végétation / système racinaire / racine adventive

Summary - Relationship between morphological characters and root lodging susceptibility of maize (Zea mays L). The relationship between morphological characters and root lodging susceptibility was studied for 12 maize hybrids. The 12 hybrids were sown in 1988 in a 3 block design with individual plots of 2 rows, $5 \mathrm{~m}$ long. This first design was used for measuring natural lodging. The 12 hybrids were also sown in a 2nd design with greater plots (4 rows $\times \mathbf{4 0}$ m) for plant sampling. Twenty four plants per hybrid were sampled on several dates. The following data were recorded: number of visible and expanded leaves, aerial dry matter per plant and number of emerged roots per internode. Natural lodging occurred on the 1st of July when plants had between 13 and 15 visible leaves. The inclination angle was measured on 50 plants per plot using the rule shown in figure 1. The 50 plants were distributed among 6 angle classes (table 1). A mean inclination angle was then calculated for each hybrid. Some lodged plants were carefully removed from the soil for detailed observation of damages and breaking points on the root system and at the base of the stem. Seven of the 12 hybrids were lodged. The mean inclination angle ranged from 0 to 19.9 degrees for the most lodged hybrid (table III). Plants were inclined from their base without any stem breakage. Detailed observation of lodged plants showed breakage points on the basal part of the roots of the upper internodes (figs 2-3; table IV). The plant sample which took place just before natural lodging showed differences between hybrids for all characters (table $\mathrm{V}$ ). The mean inclination angle was negatively correlated with the number of internodes with emerged roots $\left(r=-0.677^{*}\right)$ (table VI, fig 5). These results emphasize the role of the roots of the upper internodes on stem stability. The date of appearance of these roots, and their mechanical properties, must also be taken into account. 


\section{INTRODUCTION}

La verse en végétation du maïs est un accident généralement provoqué par des vents violents accompagnés de pluies. Elle peut apparaître pendant une période allant de la montaison à la maturité. Elle doit être distinguée des verses pathologiques apparaissant en fin de cycle, dues à la contamination de la base des plantes par des agents pathogènes éventuellement favorisée par une sénescence d'origine physiologique (Barrière, 1985). Dans les 2 cas, il peut en résulter des pertes de rendement, des difficultés de récolte et dans le cas du maïs ensilage une baisse de qualité du produit récolté. L'amélioration de ce caractère demeure une préoccupation importante des sélectionneurs.

Classiquement, la sélection pour la résistance à la verse est faite par notation du pourcentage de tiges versées en essais multilocaux. Les limites de cette méthode sont évidentes : la comparaison des génotypes n'est possible que si le climat de l'année a permis l'extériorisation du phénomène. Le classement obtenu dépend des conditions locales d'apparition de la verse. Enfin, le placement contigu des parcelles d'essai avec des variétés plus ou moins résistantes biaise en partie la mesure du fait des effets de proximité. II en résulte certaines années des difficultés pour opérer un tri du matériel végétal.

Plusieurs auteurs ont recherché d'autres méthodes d'appréciation de la résistance à la verse des génotypes. Une mesure de la force verticale nécessaire à l'arrachement des pieds a été proposée (Penny, 1981; Fincher et al, 1985). Ce test est parfois utilisé en sélection mais, d'après un travail récent, ses résultats sont faiblement corrélés à la résistance à la verse en végétation (Melchinger et al, 1986). D'autres auteurs ont recherché des corrélations entre des caractères morphologiques, mécaniques ou histologiques des plantes susceptibles d'intervenir dans la tenue de la tige et l'importance de la verse en conditions naturelles (Remison et Akinleye, 1978; Ishige et al, 1983; Helms et Compton, 1984; Adamou Amoukou, 1986; Atta, 1987; Varlet-Grancher et al, 1987). Les résultats acquis ne permettent pas encore l'utilisation effective de tels critères en sélection.

L'objectif de cet article est de présenter quelques relations observées entre des caractères mesurés sur 12 hybrides en 1988 et les résultats d'une verse intervenue 2 jours après la réalisation des mesures. Cette coïncidence entre les mesures effectuées et l'apparition de la verse est rarement obtenue.

\section{MATÉRIEL ET MÉTHODE}

La parcelle expérimentale est située sur sol limoneux profond (argile : $25,1 \%$, limon fin : $32,6 \%$, limon grossier : $34,5 \%$, sable fin : $5,4 \%$, sable grossier : $2,4 \%$, matière organique : $1,9 \%, \mathrm{pH}$ (eau) : 8,1 ; domaine expérimental INRA Colmar). Le sol est correctement pourvu en éléments chimiques $\left(\mathrm{P}_{2} \mathrm{O}_{5} \mathrm{JH}: 0,26 \%\right.$; $\mathrm{K}_{2} \mathrm{O}: 0,39 \%$ \%). La préparation du sol, le désherbage et la fertilisation ont été conduits selon les normes locales de la grande culture : labour en août 1987; préparation du sol par passage d'un chisel (15 avril 1988) et d'un vibroculteur (26 avril 1988); apport de $145 \mathrm{~kg} /$ ha de $\mathrm{N}, \mathrm{P}_{2} \mathrm{O}_{5}$ et $\mathrm{K}_{2} \mathrm{O}$ (26 avril 1988). Le semis a été effectué le 29 avril, à une densité de 130000 pieds/ ha. Un éclaircissage à 80000 pieds/ha a été réalisé le 30 mai.

Le matériel végétal utilisé était constitué de 11 hybrides non commerciaux et d'une variété de référence (Dea). L'essai a été divisé en 2 parties :

- un essai bloc à 3 répétitions sur lequel ont été effectuées les notations de verse naturelle. Chaque bloc $(18 \mathrm{~m} \times 5 \mathrm{~m})$ comprenait 12 parcelles élémentaires de 2 rangs de $5 \mathrm{~m}$, orientés nord-sud et correspondant aux 12 hybrides. Deux blocs supplémentaires non utilisés pour les notations ont été implantés de part et d'autre de l'essai pour limiter les effets de bordure;

-12 grandes parcelles (4 rangs $\times 40 \mathrm{~m}$ ) correspondant aux 12 hybrides et destinées à des prélèvements de plantes.

Un prélèvement de 24 plantes par variété a été effectué le 29 juin, soit 644,5 degrés-jours après semis en base $6{ }^{\circ} \mathrm{C}$. Les variables mesurées ont été le nombre de feuilles visibles et étalées, la matière sèche des parties aériennes et le nombre de racines adventives émises par entrenœud. Les entrenœuds ont été numérotés conformément au système de notation proposé par Girardin et al (1986). Le mésocotyle est assimilé à un entrenœud noté $E_{1}$ et le premier entrenœud portant des racines adventives est noté $E_{2}$. Une racine a été considérée comme émise dès qu'elle a percé la gaine de la feuille couvrant l'entrenœud dont elle est issue.

La verse eut lieu le $1^{\mathrm{er}}$ juillet. La vitesse maximale du vent a été de $20 \mathrm{~m} / \mathrm{s}$ d'orientation sud-ouest-nordest et la pluviométrie de $11,6 \mathrm{~mm}$. Les notations de verse ont été effectuées sur 50 plantes par parcelle élémentaire, en répartissant les pieds par classe d'inclinaison par rapport à la verticale (tableau I). Un schéma de la règle utilisée est présenté figure 1. Pour chaque parcelle on a calculé un angle moyen d'inclinaison de la façon suivante :

$$
A=\frac{1}{n} \sum_{i=0}^{i=5} n_{i} A_{i}
$$


Tableau I. Définition des classes d'inclinaison utilisées pour les notations de verse naturelle.

\begin{tabular}{cccc}
\hline $\begin{array}{c}\text { Classe } \\
\text { d'inclinaison }\end{array}$ & \multicolumn{2}{c}{$\begin{array}{c}\text { Angle par rapport à } \\
\text { la verticale (en degrés) }\end{array}$} \\
\hline & & & \\
0 & & 0 & \\
1 & ] 0 & $;$ & $6]$ \\
2 & ] 6 & $;$ & $12]$ \\
3 & ] 12 & $;$ & $24]$ \\
4 & ] 24 & $;$ & $48]$ \\
5 & ] 48 & $;$ & $90]$ \\
\hline
\end{tabular}

avec $n=$ effectif total de plantes observées, $n_{i}=$ effectif dans la classe d'inclinaison i, $A_{i}=$ angle moyen d'inclinaison de la classe $i$.

Sur 3 hybrides (numéros 2, 5 et 14) 8 plantes versées ont été prélevées avec précaution pour une observation détaillée des zones de rupture à la base des tiges et sur les racines.

Des notations de stade ont été effectuées les 18 et 25 juillet ( 881,0 et 983,6 degrés-jours après semis). À la récolte, réalisée le 21 octobre, soit 1887,5 degrés-jours après semis, le poids de grain et leur teneur en eau ont été mesurés.

\section{RÉSULTATS}

\section{Caractérisation des hybrides}

Dans le tableau II, on a indiqué pour chaque hybride le stade atteint le 18 et le 25 juillet et la te-

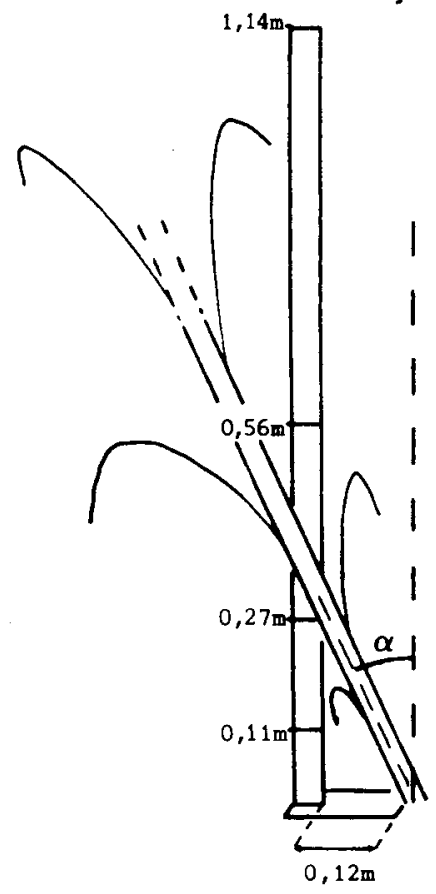

Fig 1. Règle utilisée pour les mesures d'angle d'inclinaison des plantes (d'après Duparque, 1988).
Tableau II. Stade des plantes les 18 et 25 juillet 1988 et teneur en eau des grains à la récolte. $P E$ : panicule étalé. FM : floraison mâle. SS : sortie des soies. FF : floraison femelle.

\begin{tabular}{rccc}
\hline Hybride & $\begin{array}{c}\text { Stade } \\
\text { le 18 juillet }\end{array}$ & $\begin{array}{c}\text { Stade } \\
\text { le 25 juillet }\end{array}$ & $\begin{array}{c}\text { Moyenne } \\
\text { (écart type) } \\
\text { test N et K }\end{array}$ \\
\hline & & & \\
15 & début FF & FF & $25,77(0,34) \mathrm{a}$ \\
12 & $\mathrm{PE}$ & début FF & $26,26(0,56) \mathrm{ab}$ \\
11 & $\mathrm{FM}$ & début FF & $27,54(0,53) \mathrm{bc}$ \\
13 & début FF & $\mathrm{FF}$ & $27,62(0,17) \mathrm{bc}$ \\
9 & $\mathrm{PE}$ & $\mathrm{FF}$ & $27,70(0,65) \mathrm{bc}$ \\
2 & $\mathrm{FF}$ & fin FF & $27,77(0,35) \mathrm{bc}$ \\
5 & début FF & $\mathrm{FF}$ & $28,31(1,96) \mathrm{c}$ \\
14 & début FF & $\mathrm{FF}$ & $28,43(0,72) \mathrm{c}$ \\
10 & $\mathrm{FM}$ & $\mathrm{FF}$ & $29,70(0,46) \mathrm{d}$ \\
18 & $\mathrm{PE}$ & $\mathrm{FF}$ & $30,13(0,49) \mathrm{d}$ \\
16 & $\mathrm{SS}$ & $\mathrm{FF}$ & $30,57(0,37) \mathrm{d}$ \\
8 & $\mathrm{FM}$ & $\mathrm{FF}$ & $31,11(0,54) \mathrm{d}$ \\
\hline
\end{tabular}

neur en. eau des grains à la récolte. L'humidité des grains varie de 25,8 à $31,1 \%$, l'hybride commercial Dea (2) se situant au milieu de la gamme $(27,8 \%)$. Ces résultats situent l'ensemble des hybrides étudiés dans une gamme de précocité proche de Dea.

\section{Notations de verse naturelle}

\section{Classement des hybrides}

Les résultats des notations de verse et l'angle moyen d'inclinaison calculé par hybride sont notés dans le tableau III. Les hybrides $8,10,12$, 16 et 18 n'ont pas versé. La verse la plus importante a été observée pour les hybrides 5 et 15 pour lesquels 96 et $79 \%$ des plantes ont versé avec un angle moyen d'inclinaison de 19,9 et 16,4 degrés respectivement. Si l'on considère l'ensemble des hybrides la variable angle moyen d'inclinaison ne satisfait pas aux conditions nécessaires à l'utilisation de l'analyse de variance (normalité des résidus et égalité des variances résiduelles). Ces conditions sont, par contre, satisfaites si l'on considère uniquement les hybrides ayant versé $(2,5,9,11,13,14$ et 15). Une analyse de variance conclut dans ce cas à l'existence de différences significatives entre hybrides $(F=11,72$ pour $6 \mathrm{ddl})$. On a confronté le classement des hybrides obtenu à Colmar au même classement établi en 5 lieux pour des verses intervenues à la même période (tableau 
Tableau III. Pourcentage de tiges par classe d'inclinaison et angle moyen d'inclinaison (A) calculé par hybride. (1) Source Limagrain 1988.

\begin{tabular}{|c|c|c|c|c|c|c|c|c|c|}
\hline $\begin{array}{c}\text { Classe } \\
\text { d'inclinaison }\end{array}$ & 0 & {$[0,6]$} & ] $6,12]$ & J12,24] & ] $24,48]$ & ] $48,90]$ & $\begin{array}{l}\text { A. Moyenne } \\
\text { (écart type) }\end{array}$ & Rang & $\begin{array}{c}\text { Rang en essai } \\
\text { multilocal (1) }\end{array}$ \\
\hline \multicolumn{10}{|l|}{ Hybride } \\
\hline 5 & 0,039 & 0,166 & 0,178 & 0,318 & 0,259 & 0,040 & $19,90(4,51)$ & 1 & 3 \\
\hline 15 & 0,213 & 0,188 & 0,151 & 0,150 & 0,265 & 0,033 & $16,43(5,04)$ & 2 & 1 \\
\hline 14 & 0,487 & 0,280 & 0,107 & 0,073 & 0,053 & 0 & $5,04(2,37)$ & 3 & 5 \\
\hline 11 & 0,493 & 0,233 & 0,173 & 0,053 & 0,047 & 0 & $4,90(1,89)$ & 4 & 2 \\
\hline 9 & 0,680 & 0,160 & 0,080 & 0,047 & 0,020 & 0,013 & $3,68(2,70)$ & 5 & 8 \\
\hline 2 & 0,573 & 0,333 & 0,087 & 0,007 & 0 & 0 & $1,90(0,62)$ & 6 & 4 \\
\hline 13 & 0,980 & 0,013 & 0,007 & 0 & 0 & 0 & $0,10(0,14)$ & 7 & 9 \\
\hline 10 & 1 & 0 & 0 & 0 & 0 & 0 & $0 \quad(0)$ & 8 & 6 \\
\hline 12 & 1 & 0 & 0 & 0 & 0 & 0 & (0) & 8 & 7 \\
\hline 8 & 1 & 0 & 0 & 0 & 0 & 0 & (0) & 8 & 10 \\
\hline 18 & 1 & 0 & 0 & 0 & 0 & 0 & (0) & 8 & 11 \\
\hline 16 & 1 & 0 & 0 & 0 & 0 & 0 & (0) & 8 & 12 \\
\hline
\end{tabular}

III). Les hybrides ayant fortement versé à Colmar (5 et 15) apparaissent également parmi les plus sensibles en essai multilocal. De même, les hybrides 8,16 et 18 qui n'ont pas versé à Colmar sont classés comme étant les plus résistants.

\section{Types de verse et description des zones de rupture}

Pour tous les hybrides concernés, la verse se caractérise par des plantes inclinées depuis la base. On n'observe aucune cassure de tige. L'inclinaison des pieds est dans le sens sud-ouestnord-est. L'observation détaillée de 24 pieds versés des hybrides 2,5 et 14 montre l'existence de coudes ou de ruptures sur la partie basale des racines adventives et au niveau du point d'insertion de ces racines sur la tige. On a distingué 5 cas décrits dans le tableau IV et en figure 2. Dans la figure 3 on a noté pour chaque entrenœud le pourcentage de racines concernées. Les racines les plus touchées sont celles des derniers entrenœuds ayant émis des racines avant la verse : entrenœuds 5 et 6 pour les hybrides 2 et 14, entrenœuds 4 et 5 pour l'hybride 5 . Les cas les plus fréquents sont les types 1 et 3. Les coudes ou ruptures observés au niveau du cortex entraînent une perte de rigidité de la racine sans que le cylindre central ne soit pour autant sectionné. II y a très rarement cassure totale de la racine (type 4 ou 5).

Tableau IV. Description des coudes et des ruptures sur les racines des pieds versés.

$\begin{array}{lll}\text { Type de rupture } \quad \text { Description } & \text { Photo }\end{array}$

2
La racine est coudée

fig $2 a$

La racine est coudée avec rupture du cortex

fig $2 b$

Le cortex est rompu et fendu sur une certaine longueur.

II y a décollement de celui-ci du cylindre central.

fig $2 c$

Une partie du cortex a été arrachée.Le cylindre central est à nu et éventuellement rompu un peu plus loin.

fig $2 d$

La racine est cassée ou séparée de la tige si la rupture se situe au niveau du point d'insertion. 


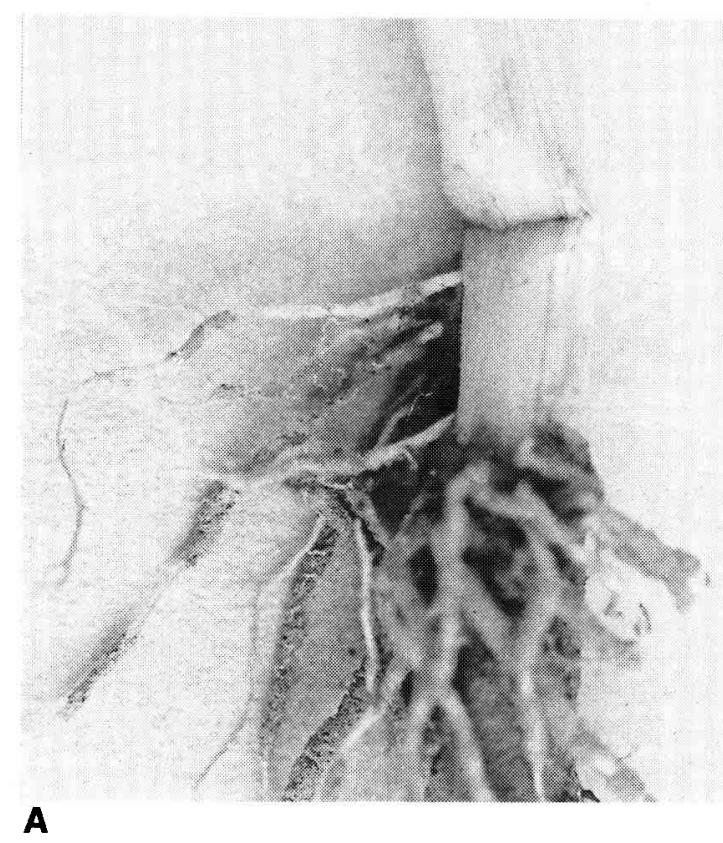

A

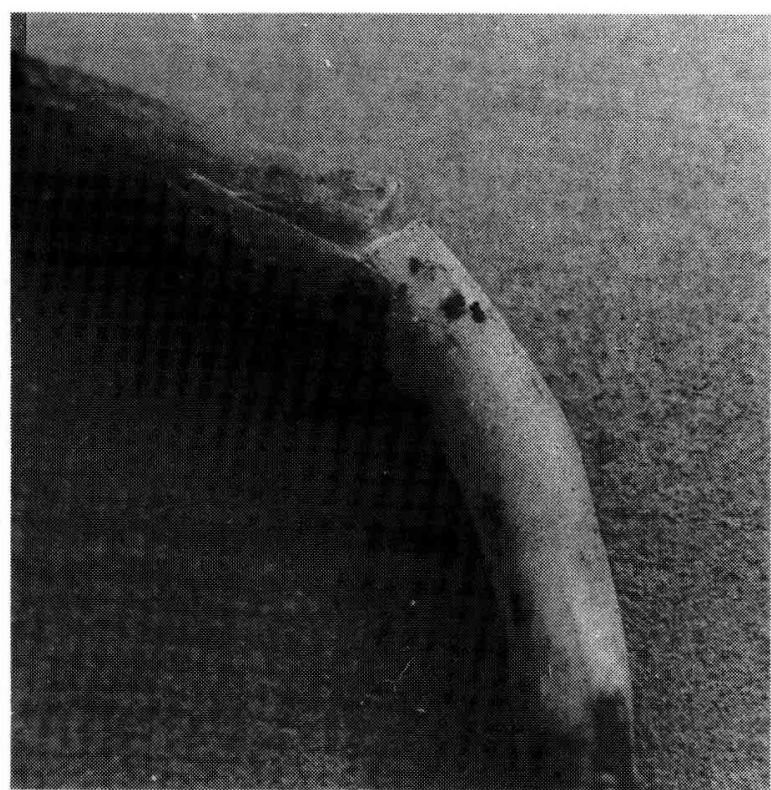

C

Fig 2. Coudes et ruptures observés sur les racines des pieds versés. a: type 1. b: type 2. c: type 3. d: type 4.

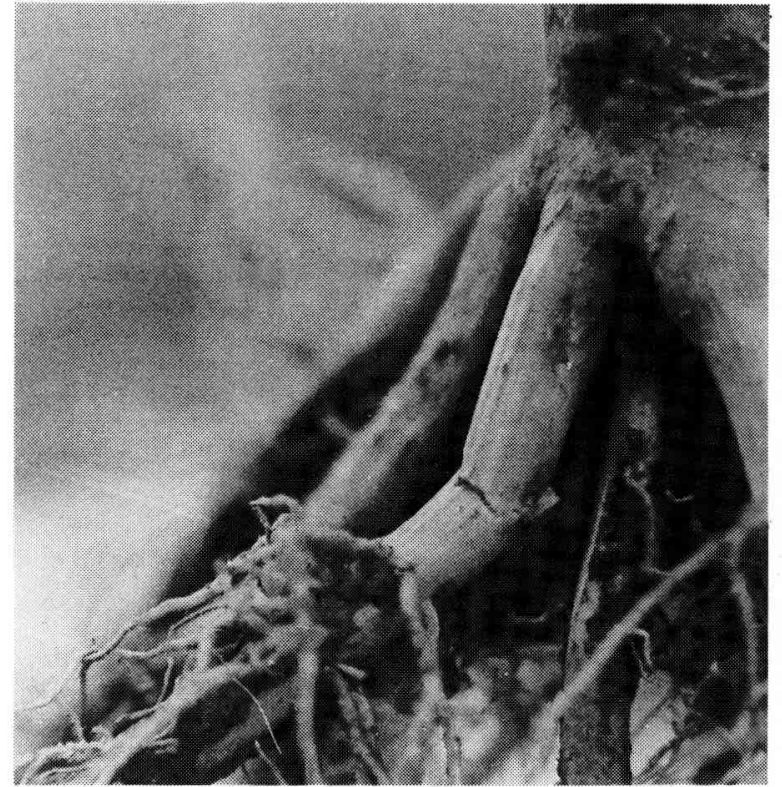

$\mathbf{B}$



D

\section{Résultats du prélèvement du 29 juin 1988}

Les valeurs moyennes par hybride pour chacune des variables mesurées et les corrélations significatives entre variables sont consignées dans les tableaux V et VI. II apparaît des différences entre hybrides pour tous les caractères mesurés. Le stade des plantes lors du prélèvement varie de 12,9 à 14,7 feuilles visibles. II n'est pas corrélé à la teneur en eau des grains à la récolte $(r=$ $0,394)$. Le poids de matière sèche aérienne varie de 24,9 (hybride 11) à 42,8 g par plante (hybride 14). Il est corrélé au nombre de feuilles visibles $(r=0,709)$. Le nombre total de racines émises varie de 18,1 (hybride 2) à 30,2 (hybride 16). Sur les premiers entrenœuds $\left(E_{2}\right.$ à $\left.E_{5}\right)$, les nombres de racines sont corrélés positivement. Le nombre moyen d'entrenœuds portant des racines allongées varie de 5,2 (hybride 5) à 6,8 (hybrides 13, 16 et 18). Dans ce dernier cas, cela signifie que la plupart des plantes portent déjà des racines sur l'entrenœud 7. Le nombre d'entrenœuds portant des racines est corrélé positivement au nombre de feuilles visibles $(r=0,752)$. Il est corrélé négativement au nombre de racines sur les premiers entrencuds $\left(E_{3}, E_{4}\right.$ et $\left.E_{5}\right)$. Cette tendance est illustrée figure 4. L'hybride 5 en particulier a un nombre élevé 



Fig 3. Pourcentage de racines sur lesquelles on observe des ruptures, par entrenœud et par type de rupture.

de racines sur les entrenœuds $E_{2}$ à $E_{5}$ mais le nombre d'entrenœuds portant des racines est faible.

Dans le tableau VI, on a noté la valeur des coefficients de corrélation entre l'angle d'inclinaison moyen calculé $(A)$ et chacune des variables mesurées lors du prélèvement. On observe une corrélation négative entre le nombre d'entrenœuds portant des racines allongées et l'angle moyen d'inclinaison. La relation entre ces 2 variables est présentée en figure 5 . Sur l'hybride 5 qui a fortement versé le nombre d'entrenœuds portant des racines est faible. À l'autre extrême les hybrides 13,16 et 18 pour lesquels le nombre d'entrenœuds ayant émis des racines est élevé n'ont pas versé. Sur les autres hy-



Fig 4. Relation entre le nombre total d'entrenœuds portant des racines et le nombre de racines sur les entrenœuds $E_{2}$ à $\mathrm{E}_{5}$.

brides, on observe de fortes différences au niveau de l'importance de la verse alors que les nombres d'entrenœuds portant des racines sont très proches. Cela suggère l'intervention probable d'autres caractères dans l'importance de la verse.

On observe une corrélation positive entre l'angle d'inclinaison et le nombre de racines sur les premiers entrenœuds. On peut penser que cela résulte de la corrélation négative déjà signalée entre le nombre de racines sur les premiers entrenœuds et le nombre d'entrenœuds portant des racines (tableau VI et fig 4). Les coefficients de corrélation partielle entre l'angle d'inclinaison et le nombre de racines sur les premiers entrenœuds sont de fait très faibles.

\section{DISCUSSION ET CONCLUSION}

Les résultats précédents montrent le rôle important des racines des entrenœuds supérieurs par rapport à la résistance à la verse. Les hybrides 13,16 et 18 qui portaient déjà des racines sur l'entrenœud 7 n'ont pas versé. À l'inverse l'hy-

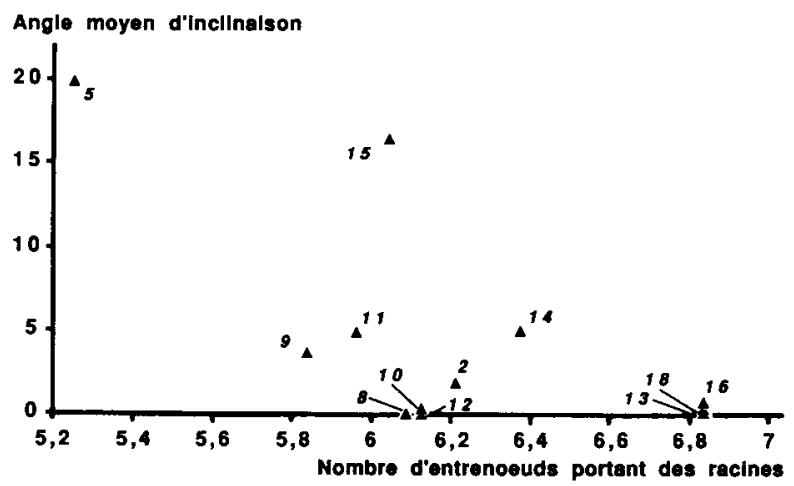

Fig 5. Relation entre l'angle d'inclinaison calculé et le nombre d'entrenœuds portant des racines. 
Tableau V. Résultats du prélèvement du 29 juin 1988. nfv : nombre de feuilles visibles. ms : matière sèche aérienne par plante. $E_{i}$ : nombre de racines adventives sur l'entrencud i. ntra: nombre total de racines adventives. nbent : nombre d'entrenœuds portant des racines. Moyennes, écarts- type, résultats du test de Newman et Keuls.

\begin{tabular}{|c|c|c|c|c|c|c|c|c|c|c|}
\hline Hybric & de $\mathrm{nfv}$ & ms (en g) & $E_{2}$ & $E_{3}$ & $E_{4}$ & $E_{5}$ & $E_{6}$ & $E_{7}$ & ntra & nbent \\
\hline 2 & $\begin{array}{c}13,92 \mathrm{bc} \\
(0,13)\end{array}$ & $\begin{array}{c}33,70 \text { bcd } \\
(3,21)\end{array}$ & $\begin{array}{c}3,37 \quad c \\
(0,38)\end{array}$ & $\begin{array}{c}3,04 \\
(0,33)\end{array}$ & $\begin{array}{cc}2,50 & f \\
(0,52) & \end{array}$ & $\begin{array}{c}3,25 \text { ef } \\
(0,32)\end{array}$ & $\begin{array}{c}4,92 \\
(1,06)\end{array} \quad C$ & $\begin{array}{l}1,04 \mathrm{c} \\
(1,44)\end{array}$ & $\begin{array}{c}18,12 \\
(1,64)\end{array}$ & $\begin{array}{c}6,21 \text { bc } \\
(0,37)\end{array}$ \\
\hline 5 & $\begin{array}{l}13,00 \mathrm{~d} \\
(0,27)\end{array}$ & $\begin{array}{c}32,94 \\
(3,07)\end{array}$ & $\begin{array}{c}4,04 \\
(0,25)\end{array}$ & $\begin{array}{c}4,83 \\
(0,34)\end{array}$ & $\begin{array}{c}4,96 \\
(0,25)\end{array} \quad a$ & $\begin{array}{c}6,92 \\
(0,90)\end{array}$ & $\begin{array}{c}2,12 \\
(2,67)\end{array}$ & $\begin{array}{c}0,00 \mathrm{c} \\
(0,00)\end{array}$ & $\begin{array}{c}22,87 \mathrm{~cd} \\
(2,30)\end{array}$ & $\begin{array}{l}5,25 d \\
(0,27)\end{array}$ \\
\hline 8 & $\begin{array}{c}13,97 \mathrm{bc} \\
(0,41)\end{array}$ & $\begin{array}{l}38,98 \text { abcd } \\
(9,32)\end{array}$ & $\begin{array}{c}4,08 \text { a } \\
(0,13)\end{array}$ & $\begin{array}{c}3,68 \text { bcd } \\
(0,36)\end{array}$ & $\begin{array}{c}3,81 \\
(0,44)\end{array}$ & $\begin{array}{c}4,60 \\
(0,40)\end{array}$ & $\begin{array}{c}8,14 \\
(1,17)\end{array}$ & $\begin{array}{l}0,33 \mathrm{c} \\
(0,54)\end{array}$ & $\begin{array}{c}24,64 \quad c \\
(1,23)\end{array}$ & $\begin{array}{l}6,08 \text { bc } \\
(0,13)\end{array}$ \\
\hline 9 & $\begin{array}{l}12,87 \mathrm{~d} \\
(0,26)\end{array}$ & $\begin{array}{c}31,20 \\
(3,14)\end{array}$ & $\begin{array}{l}3,92 \mathrm{ab} \\
(0,26)\end{array}$ & $\begin{array}{c}3,92 \\
(0,38)\end{array}$ & $\begin{array}{c}3,96 \quad b \\
(0,33)\end{array}$ & $\begin{array}{cc}5,17 & b \\
(0,56) & \end{array}$ & $\begin{array}{l}6,54 \mathrm{abc} \\
(0,84)\end{array}$ & $\begin{array}{l}0,00 \mathrm{c} \\
(0,00)\end{array}$ & $\begin{array}{c}23,50 \mathrm{~cd} \\
(0,85)\end{array}$ & $\begin{array}{l}5,83 \quad c \\
(0,20)\end{array}$ \\
\hline 10 & $\begin{array}{l}13,37 \mathrm{~cd} \\
(0,68)\end{array}$ & $\begin{array}{c}30,64 \\
(6,87)\end{array}$ de & $\begin{array}{l}3,83 \mathrm{ab} \\
(0,13)\end{array}$ & $\begin{array}{l}3,71 \text { bcd } \\
(0,29)\end{array}$ & $\begin{array}{l}3,54 \text { bcd } \\
(0,29)\end{array}$ & $\begin{array}{l}3,71 \text { def } \\
(0,40)\end{array}$ & $\begin{array}{l}7,12 \text { abc } \\
(1,18)\end{array}$ & $\begin{array}{l}0,62 \mathrm{c} \\
(0,83)\end{array}$ & $\begin{array}{c}22,54 \mathrm{~cd} \\
(1,83)\end{array}$ & $\begin{array}{l}6,12 b c \\
(0,14)\end{array}$ \\
\hline 11 & $\begin{array}{l}12,83 \mathrm{~d} \\
(0,72)\end{array}$ & $\begin{array}{c}24,92 \\
(4,85)\end{array}$ & $\begin{array}{l}3,87 \text { ab } \\
(0,14)\end{array}$ & $\begin{array}{l}3,21 \text { cde } \\
(0,33)\end{array}$ & $\begin{array}{l}3,21 \text { cde } \\
(0,25)\end{array}$ & $\begin{array}{l}4,00 \text { cde } \\
(0,32)\end{array}$ & $\begin{array}{c}5,62 \text { bc } \\
(1,69)\end{array}$ & $\begin{array}{l}0,29 \mathrm{c} \\
(0,71)\end{array}$ & $\begin{array}{c}20,21 \text { de } \\
(1,81)\end{array}$ & $\begin{array}{c}5,96 \quad c \\
(0,25)\end{array}$ \\
\hline 12 & $\begin{array}{l}14,00 \text { bc } \\
(0,45)\end{array}$ & $\begin{array}{l}38,49 \text { abcd } \\
(4,03)\end{array}$ & $\begin{array}{c}4,08 \\
(0,20)\end{array}$ & $\begin{array}{l}3,62 \text { bcde } \\
(0,21)\end{array}$ & $\begin{array}{l}3,67 \text { bc } \\
(0,38)\end{array}$ & $\begin{array}{l}4,29 \mathrm{~cd} \\
(0,43)\end{array}$ & $\begin{array}{l}6,87 \mathrm{abc} \\
(0,56)\end{array}$ & $\begin{array}{l}0,46 \mathrm{c} \\
(0,78)\end{array}$ & $\begin{array}{c}23,00 \mathrm{~cd} \\
(0,91)\end{array}$ & $\begin{array}{l}6,12 \text { bc } \\
(0,14)\end{array}$ \\
\hline 13 & $\begin{array}{l}13,87 \text { bc } \\
(0,41)\end{array}$ & $\begin{array}{c}31,39 \text { de } \\
(1,66)\end{array}$ & $\begin{array}{l}3,83 \mathrm{ab} \\
(0,13)\end{array}$ & $\begin{array}{c}3,12 \\
(0,26)\end{array}$ de & $\begin{array}{l}3,04 \text { de } \\
(0,10)\end{array}$ & $\begin{array}{c}3,42 \\
(0,41)\end{array}$ & $\begin{array}{c}5,54 \text { bc } \\
(0,53)\end{array}$ & $\begin{array}{l}3,58 \mathrm{~b} \\
(1,79)\end{array}$ & $\begin{array}{c}22,54 \mathrm{~cd} \\
(1,75)\end{array}$ & $\begin{array}{c}6,83 \text { a } \\
(0,20)\end{array}$ \\
\hline 14 & $\begin{array}{c}14,71 \text { a } \\
(0,25)\end{array}$ & $\begin{array}{c}42,85 \\
(5,12)\end{array}$ & $\begin{array}{l}3,54 \text { bc } \\
(0,25)\end{array}$ & $\begin{array}{c}3,17 \\
(0,54)\end{array}$ de & $\begin{array}{l}2,46 \quad f \\
(0,33)\end{array}$ & $\begin{array}{c}3,08 \\
(0,30)\end{array}$ & $\begin{array}{l}6,33 \text { abc } \\
(1,34)\end{array}$ & $\begin{array}{l}3,12 \mathrm{~b} \\
(2,82)\end{array}$ & $\begin{array}{l}21,71 \mathrm{~cd} \\
(3,85)\end{array}$ & $\begin{array}{c}6,37 \text { b } \\
(0,26)\end{array}$ \\
\hline 15 & $\begin{array}{l}13,42 \mathrm{~cd} \\
(0,30)\end{array}$ & $\begin{array}{c}41,21 \\
(3,98)\end{array}$ & $\begin{array}{l}3,62 \text { bc } \\
(0,14)\end{array}$ & $\begin{array}{c}3,79 \\
(0,25)\end{array}$ & $\begin{array}{l}2,92 \text { ef } \\
(0,49)\end{array}$ & $\begin{array}{l}3,62 \text { def } \\
(0,26)\end{array}$ & $\begin{array}{l}7,54 \text { ab } \\
(1,70)\end{array}$ & $\begin{array}{l}0,25 \mathrm{c} \\
(0,61)\end{array}$ & $\begin{array}{c}21,75 \mathrm{~cd} \\
(1,80)\end{array}$ & $\begin{array}{l}6,04 \text { bc } \\
(0,10)\end{array}$ \\
\hline 16 & $\begin{array}{c}14,37 a b \\
(0,38)\end{array}$ & $\begin{array}{l}34,91 \text { abcd } \\
(2,15)\end{array}$ & $\begin{array}{l}3,75 a b \\
(0,22)\end{array}$ & $\begin{array}{l}3,42 \text { bcde } \\
(0,38)\end{array}$ & $\begin{array}{l}3,04 \text { de } \\
(0,19)\end{array}$ & $\begin{array}{l}3,79 \text { def } \\
(0,37)\end{array}$ & $\begin{array}{c}8,25 \\
(1,07)\end{array}$ a & $\begin{array}{l}7,92 \mathrm{a} \\
(1,80)\end{array}$ & $\begin{array}{c}30,17 \text { a } \\
(1,79)\end{array}$ & $\begin{array}{c}6,83 \text { a } \\
(0,13)\end{array}$ \\
\hline 18 & $\begin{array}{c}14,71 \text { a } \\
(0,40)\end{array}$ & $\begin{array}{l}40,49 \text { abc } \\
(3,08)\end{array}$ & $\begin{array}{l}3,83 a b \\
(0,26)\end{array}$ & $\begin{array}{l}3,62 \text { bcde } \\
(0,31)\end{array}$ & $\begin{array}{l}3,08 \text { de } \\
(0,20)\end{array}$ & $\begin{array}{l}3,96 \text { cde } \\
(0,43)\end{array}$ & $\begin{array}{l}7,04 \text { abc } \\
(0,83)\end{array}$ & $\begin{array}{l}6,37 \mathrm{a} \\
(1,88)\end{array}$ & $\begin{array}{c}27,92 \text { b } \\
(1,34)\end{array}$ & $\begin{array}{c}6,83 \text { a } \\
(0,20)\end{array}$ \\
\hline
\end{tabular}

bride 5, qui ne portait pas encore de racines sur les entrenœuds $E_{6}$ et $E_{7}$ a fortement versé, bien que le nombre total de racines adventives émises soit comparable à celui d'autres hybrides (tableau V). L'importance du nombre de racines sur les entrenœuds supérieurs par rapport à la résistance à la verse a été signalée par d'autres auteurs (Atta, 1987; Duparque, 1988). Les observations faites dans cet essai soulignent la nécessité de considérer non seulement le nombre de racines sur ces entrenœuds mais aussi la précocité d'émission de ces racines, en particulier pour rendre compte de la sensibilité à la verse antéfloraison.

Le nombre d'entrenœuds portant des racines allongées ne suffit pas cependant à rendre compte des différences de sensibilité à la verse observées pour certains hybrides (hybrides 11 et 15 , par exemple, fig 4). D'autres caractères morphologiques ou des propriétés mécaniques des plantes sont évidemment susceptibles d'intervenir dans le mécanisme de sensibilité à la verse. La verse résulte a priori d'un déséquilibre entre les parties aériennes, dont dépend la prise au vent, et l'ancrage des plantes. Les caractères impliqués concernent donc à la fois l'appareil aérien (hauteur des plantes, répartition et importance de la surface foliaire, hauteur et masse de l'épi pour les verses post-floraison), les propriétés mécaniques de la tige et le système racinaire (nombre de racines par entrenœud, hauteur d'insertion par rapport au sol, diamètre et angle d'émission, présence ou non de ramifications). Les données bibliographiques montrent d'ailleurs la multiplicité des caractères en jeu. L'évolution de l'ancrage doit être étudiée en regard de l'évolution des parties aériennes. Des différences sur ces caractères (hauteur des plantes, présence ou non de racines secondaires sur les derniers niveaux de racines adventives émis, etc) peuvent expliquer les différences de comportement observées entre des hybrides portant sensiblement le même nombre de niveaux de racines.

L'observation détaillée des pieds versés a montré l'existence de coudes ou de ruptures sur la partie basale des racines des entrenœuds supérieurs. Ce type d'observation est rarement ef- 
Tableau VI. Matrice des corrélations entre les variables mesurées. $W$ : teneur en eau du grain à la récolte. $A$ : angle moyen d'inclinaison calculé. ${ }^{*} 0,01<P<0,05 .{ }^{* \star} 0,001<P<0,01 .{ }^{* \star *} P<0,001$.

\begin{tabular}{|c|c|c|c|c|c|c|c|c|c|c|c|c|}
\hline & nfv & $\mathrm{ms}$ & $E_{2}$ & $E_{3}$ & $E_{4}$ & $E_{5}$ & $\mathrm{E}_{6}$ & $E_{7}$ & ntra & nbent & w & A \\
\hline $\begin{array}{l}\mathrm{nfv} \\
\mathrm{ms}\end{array}$ & $\begin{array}{l}1 \\
0,709^{\star \star}\end{array}$ & 1 & & & & & & & & & & \\
\hline $\begin{array}{l}E_{2} \\
E_{3} \\
E_{4}\end{array}$ & & & $\begin{array}{l}1 \\
0,808^{* *}\end{array}$ & $\begin{array}{l}1 \\
0,884^{* * *}\end{array}$ & 1 & & & & & & & \\
\hline$E_{5}$ & & & $0,666^{*}$ & $0,905^{\star \star \star}$ & $0,950^{\star \star \star}$ & 1 & & & & & & \\
\hline $\begin{array}{l}E_{6} \\
E_{7}\end{array}$ & $0,723^{\star \star}$ & & & & & & 1 & 1 & & & & \\
\hline ntra & & & & & & & & $0,742^{* *}$ & 1 & & & \\
\hline nbent & $0,752^{\star *}$ & & & $-0,695^{*}$ & $-0,694^{*}$ & $-0,732$ & & $0,830^{\star * *}$ & & 1 & & \\
\hline$w$ & & & & & & & & & $0,624^{*}$ & & 1 & \\
\hline$A$ & $-0,470$ & 0,068 & $-0,013$ & $0,659^{*}$ & 0,393 & 0,540 & $-0,558$ & $-0,399$ & $-0,265$ & $-0,677^{*}$ & $-0,429$ & 1 \\
\hline
\end{tabular}

fectué sur des pieds versés naturellement et peu de données existent à ce sujet. Le fait que des ruptures apparaissent principalement sur les racines des entrenœuds supérieurs est compréhensible, compte tenu de leur position par rapport au sol et de leur rigidité élevée. Les racines des premiers entrenœuds, plus proches de la base de la tige sont a priori moins sollicitées et elles sont également plus souples. Les ruptures observées sur les racines des entrenœuds supérieurs concernent uniquement la partie corticale des racines. La perte de rigidité qui en résulte permet une inclinaison des tiges sans qu'il y ait pour autant cassure totale des racines. Ces observations soulignent la nécessité d'étudier les propriétés mécaniques de ces racines, et donc les caractères morphologiques et biochimiques qui les déterminent.

\section{RÉFÉRENCES}

Adamou Amoukou I (1986) Variabilité génétique du système racinaire dans un diallèle de 9 lignées de maïs sensibles ou résistantes à la verse en végétation. Mémoire ENSA Rennes, $57 \mathrm{p}$

Atta $S$ (1987) Étude des relations lignées-hybrides pour la sensibilité à la verse en végétation chez le maïs fourrage. Mémoire ENSA Rennes, $43 p$

Barrière $Y$ (1985) Aspects physiologiques de la pourriture des tiges du maïs. Agronomie 5, 361-368

Duparque M (1988) Étude des verses racinaires naturelles du maïs précoce (Zea mays $L$ ) dans un ré- seau multilocal et recherche de caractères morphologiques associés. Mémoire ISAB, $91 p$

Fincher RR, Darrah LL, Zuber MS (1985) Root development in maize as measured by vertical pulling resistance. Maydica 30, 383-394

Girardin P, Jordan MO, Picard D, Trendel R (1986) Harmonisation des notations concernant la description morphologique d'un pied de maïs (Zea mays L). Agronomie 6, 873-875

Helms TC, Compton WA (1984) Ear height and weight as related to stalk lodging in maize. Crop Sci 24 , 923-924

Ishige T, Yamada M, Shiga T (1983) Screening for resistance to lodging based upon the discriminant function value in maize and biometrical analysis for genetical components. Bull Natl Inst Agric Sci, Jpn, Ser D 35, 125-152

Melchinger AE, Geiger HH, Schmidt GA (1986) Vertical root-pull resistance and its relationship to root lodging and forage traits in early maturing european inbred lines and F1 hybrids of maize. Maydica 31, 335-348

Penny LH (1981) Vertical-pull resistance of maize inbreds and their testcrosses. Crop Sci 21, 237-240

Remison SU, Akinleye D (1978) Relationship between lodging, morphological characters and yield of varieties of maize (Zea mays L). J Agric Sci Camb 91, 633-638

Varlet-Grancher C, Derieux M, Jordan MO, Girardin P, Picard D (1987) Rythme d'apparition des racines primaires du maîs (Zea mays L). II. Variations observées sur quelques génotypes. Agronomie 7, 695702 